und Erhaltungstherapie zugrunde gelegt. Empfohlen werden bei Budesonidhaltigen Generika akut täglich $0,4 \mathrm{mg}$, später 0,2 $\mathrm{mg}$ - beim Mometason-Spray sind es nur 0,2 bzw. $0,1 \mathrm{mg}$, entsprechend einer halb so großen Hubzahl. Dieser Fehler und die daraus resultierenden Abweichungen von den definierten Tagesdosen im Versorgungsalltag wirken sich gravierend auf die Kosten aus.

Verbrauch im Vergleich: In der Studie wurde der tatsächliche Verbrauch von Mometason und Budesonid überdies auf Basis von Real-life-Verordnungsdaten im Zeitraum Oktober 2004 bis September 2008 ausgewertet. Die KostenNutzen-Bewertung unter Praxisbedingungen fiel dabei für das Mometasonhaltige Nasenspray bei HNO-Ärzten deutlich günstiger aus als für die Budesonid-haltige Alternative: 24,70 Euro versus 34,04 Euro durchschnittliche Therapiekosten pro Jahr bei gesetzlich
Versicherten. Im Vergleich brauchten Patienten mit allergischer Rhinitis von dem modernen Präparat aufgrund der besseren Wirksamkeit nur etwa halb so viele Hübe wie von dem generischen Präparat. Die höhere Effizienz belegt der Gesamtverbrauch: Der Unterschied erwies sich nach 730 Tagen mit einem Minderverbrauch von 360 Hüben bei HNO-Patienten als signifikant (Allgemeinpraxen: -236 Hübe). Die geringeren Verordnungsmengen beim Einsatz des modernen Glukokortikoidsprays zeigten sich konsistent über alle saisonalen und perennialen Zeiträume hinweg. Zusätzlich weist die Analyse auch anhand geringerer Folgeverordnungen eine höhere Wirtschaftlichkeit für das moderne Spray aus. Basierend auf den ermittelten tatsächlichen Verbrauchsmengen der Substanzen ist aus der Perspektive der Gesetzlichen Krankenversicherung kein Kostenvorteil von Budesonid gegenüber Mometason abzuleiten, fassen die Autoren der Studie zusammen.

\section{Tatsachen kontra Theorie}

Die Empfehlung von theoretisch angeblich „wirtschaftlichen Generika“ ist nach diesen Ergebnissen bei allergischer Rhinitis nicht zwangsläufig günstiger als die Verordnung moderner Glukokortikoidpräparate, da Ärzte und Patienten sich natürlich nicht an theoretischen definierten Tagesdosen, sondern an den tatsächlich erforderlichen Sprühstößen, d.h. den sogenannten konkret verordneten Tagesdosen, orientieren. Die wichtigsten Aussagen der Studie sind eine gute Grundlage für die Argumentation bei Androhung eines Arzneimittelregresses nach Verordnung von modernen nasalen Glukokortikoiden. Weitergehende Informationen und die Originalpublikation der Studie können Sie über die ÄDAGeschäftsstelle beziehen.

Professor Dr. Ludger Klimek, Wiesbaden

\title{
Lokale Antikörper als Ursache einer allergischen Rhinitis
}

\section{Zwischen einer allergischen und einer nicht allergischen Rhinitis zu unterscheiden, ist das tägliche Brot in der Praxis des Allergo- logen. Zur Sonderform der rein lokalen allergischen Rhinits gibt es neue Erkenntnisse.}

$R_{\text {deas }}^{\text {h }}$ hinitis ist eine häufige Erkrankung, deren Inzidenz in der westlichen Welt auf 20-40\% der Bevölkerung eingeschätzt wird. Eine nicht allergische Rhinitis ist definiert durch den fehlenden Nachweis von Sensibilisierungen, was sich in der Praxis meist als negativer Hauttest sowie negativer serologischer IgE-Nachweis manifestiert. Histologische Untersuchungen der nasalen Mukosa von Patienten mit allergischer Rhinitis zeigten eine Infiltration mit TZellen, Eosinophilen, Makrophagen und Mastzellen, was auf eine lokale Antikörperbildung hinweist. Diese Annahme erfuhr Bestätigung, als im Nasensekret IgE-Antikörper nachgewiesen wurden.
Anfang der 70er Jahre fielen dann erstmals Patienten auf, bei denen die allergologische Anamnese für ein Allergen als Auslöser der Rhinitis sprach, entsprechende Hauttests und serologische Bestimmungen aber negativ ausfielen. Die nasale Provokation mit dem verdächtigten Allergen fiel hingegen positiv aus. Am Vorkommen allergischer Rhinopathien infolge ausschließlich lokaler Antikörperbildung war demnach kein Zweifel mehr möglich. Nach den bisherigen Untersuchungen sind häufig Hausstaubmilben, seltener Pollen die Krankheitsauslöser. Im Prinzip kommt jedoch jedes Aeroallergen als Auslöser infrage $[1,2]$.

Was die Therapieoptionen betrifft, so sollte bei diesen Patienten die sym- ptomatische Behandlung durch eine entsprechende Allergenkarenz ergänzt werden. Eine Pilotstudie zeigte, dass auch eine Hyposensibilisierung wirksam sein kann [3].

\section{Fazit}

In der Praxis ist für die Diagnostik einer lokalen allergischen Rhinitis die Anamnese wegweisend. Legt diese einen Bezug zwischen einem Allergen und der Rhinitis nahe und fallen entsprechende Hauttests negativ aus, sollte auf jeden Fall nasal provoziert werden. Verläuft dieser Test positiv, so ist der Nachweis eines nasalen allergischen Geschehens erbracht und somit auf Allergenkarenz zu achten.

Dr. Dieter Bruchhausen, Wuppertal

\section{Literatur}

1. Rondón C, Canto G, Blanca M. Local allergic rhinitis: a new entity, characterization and further studies. Curr Opin Allergy Clin Immunol 2010; 10: 1-7

2. Khan DA. Allergic rhinitis with negative skin tests. Does it exist? Allergy Asthma Proc 2009; 30: 465-9

3. Kahlert S, Buschfort R, Sennekamp J. Hyposensibilisierung Hauttest-negativer Allergien. Allergologie 1990; 13: 76 